\title{
Answer to Quiz: SBA
}

\author{
(www.medicinecpd.co.uk)
}

\section{Answer: a}

The increasing incidence and prevalence of PHPT is due to the widespread use of multichannel autoanalysers finding raised calcium in patients who are asymptomatic at the time of detection (75-80\%). Therefore medical management, chiefly careful lifelong surveillance, is a reasonable alternative to surgery. The strongest indication for surgery in this patient would be hypercalciuria, even though this does not imply impaired renal function or correlate particularly well with risk of renal stone(s). Followup studies in patients below age 50 show a risk of progression over 10 years, leading to surgery of $27 \%$. The risk of parathyroid carcinoma is minimal $(<0.5 \%)$. Bone mineral density (BMD) should be measured and monitored; only a $\mathrm{T}$ score $<2.5$ would be an indication for surgery. Transient post-operative hypoparathyroidism and hypocalaemia due to 'hungry bones' is relatively common and treatable but permanent hypoparathyroidism is rare $(<2 \%)$; though reassuring, this, in itself, is not an indication for surgery.

\section{Answer:e}

One of the much taught peripheral clinical manifestations is found in less than $25 \%$ cases of IE. Blood cultures must be taken before initiating empirical treatment; ideally three sets from three different sites in the first 24 hours unless the patient is acutely unwell, when this is reduced to 2 hours. Currently Staphylococcus aureus outnumber streptococci as the leading cause. Removal of an infected pacing system or an intra cardiac prosthesis require extension of treatment from the usual 4 weeks to 6 weeks. Ceftriaxone or teicoplanin given IM or IV once daily for 2 weeks for a sensitive streptococcal infection is equivalent to more prolonged conventional treatment and can be used in a domiciliary setting with the appropriate clinical and social infrastructure.

\section{Answer.c}

HUS is a dangerous complication, induced by toxin, of infection with E.Coli 0157, the enterohaemorrhagic form of E.Coli (EHEC), or with Shigella dysenteriae. Complications with Campylobacter jejuni are rare: reactive arthritis after a few weeks and GBS in less than 1 in 1000 cases. Bacteraemia may result in osteomyelitis particularly in the immune compromised. Recently an immunoproliferative small intestinal disease (IPSID) form of lymphoma has been described which, in its early stages, may respond to antimicrobial therapy. 